\title{
Combined Analysis of the High-Energy Cosmic Neutrino Flux at the IceCube Detector
}

\section{The IceCube Collaboration}

http://icecube.wisc.edu/collaboration/authors/icrc15_icecube

E-mail: lars.mohrmann@desy.de

\begin{abstract}
With the discovery of a high-energy cosmic neutrino flux, the IceCube Neutrino Observatory, located at the geographical South Pole, has opened the field of neutrino astronomy. While evidence for extraterrestrial neutrinos has been found in multiple searches, it was not yet possible to identify their sources; they appear as an isotropic excess. Nevertheless, it is possible to constrain the properties of the sources by measuring the energy spectrum and the flavor composition of the flux, which has been done in several analyses. Typically, these analyses concentrate on specific event classes, such as events with interaction vertices inside the instrumented volume or throughgoing, $v_{\mu}$-induced tracks from the Northern hemisphere. Here, we present the latest results from a global analysis, combining the event samples of multiple individual searches, thus covering all detection channels. We derive the energy spectrum and flavor composition of the cosmic neutrino flux in the $\mathrm{TeV}-\mathrm{PeV}$ energy range. In addition, we show projected sensitivities to the cosmic neutrino flux that can be obtained with further data in the future.
\end{abstract}

Corresponding author: L. Mohrmann

DESY, Platanenallee 6, D-15738 Zeuthen, Germany

The 34th International Cosmic Ray Conference,

30 July - 6 August, 2015

The Hague, The Netherlands 


\section{Introduction}

The discovery of a cosmic neutrino flux in the TeV-PeV energy range at the IceCube Neutrino Observatory [1] has triggered a number of follow-up investigations [2, 3, 4, 5]. Each of these studies was focused on a particular aspect of the flux. Recently, a combined analysis has obtained the most accurate general characterization of the flux so far [6], based on three of the mentioned studies $[2,3,4]$ and on previous searches performed with data taken during the construction phase of the IceCube detector [7, 8, 9]. Here, we present the latest results from this analysis, taking into account new data $[10,11,12]$. In addition, we show projected constraints on the properties of the cosmic neutrino flux that can be obtained with more data in the future.

\section{Motivation \& Expectations}

Cosmic neutrinos are produced in interactions of high-energy cosmic rays with matter or radiation [13]. It is expected that such interactions frequently occur within, or close to, the as yet unknown acceleration sites of the cosmic rays [14]. Because neutrinos are neutral and weakly interacting, they can travel unhindered to the Earth, carrying with them unique information about the environments they originate from. Candidate sites for high-energy neutrino emission include active galactic nuclei, gamma-ray bursts, and starburst galaxies, but also objects within our Galaxy, such as supernova remnants or pulsar wind nebulae [15].

Ultimately, the aim of neutrino astronomy is to resolve, and thus identify, individual sources of high-energy neutrinos. However, this was not yet possible for the observed cosmic neutrino flux; the arrival directions of the neutrinos are consistent with an isotropic flux [2, 10]. Nevertheless, it is possible to constrain the properties of the sources by measuring general characteristics of the flux, such as its energy spectrum and its composition of neutrino flavors [16, 17].

The expected energy spectrum of the cosmic neutrino flux depends on the energy spectrum of the primary cosmic rays as well as on the type of interactions and the environments of the source. If the Fermi shock acceleration mechanism is responsible for the acceleration of the cosmic rays, a power law energy spectrum with spectral index close to -2 is expected [18]. If, in addition, the neutrinos are created in interactions of cosmic rays with ambient matter (i.e. pp-interactions), the energy spectrum of the secondary neutrinos approximately follows that of the primary cosmic rays. The energy spectrum of neutrinos created in interactions of cosmic rays with photons (i.e. $\mathrm{p} \gamma$-interactions) will depend on the target photon field [16]. A power law spectrum with index -2 has served as a popular benchmark scenario in the past.

The majority of cosmic neutrinos are expected to be created in the decay of charged pions $\left(\pi \rightarrow \mu+v_{\mu}\right)$ and the subsequent decay of the muons $\left(\mu \rightarrow e+v_{e}+v_{\mu}\right)$. In this scenario, the initial composition of neutrino flavors is $v_{e}: v_{\mu}: v_{\tau}=1: 2: 0$. During propagation, the composition is transformed due to neutrino oscillations, leading to an expected composition of approximately $1: 1: 1$ at the Earth [17]. In environments with a large magnetic field, the flux of neutrinos from muon decay is suppressed; in the limiting case the flavor composition becomes $0: 1: 0$ (approximately $1: 1.8: 1.8$ at Earth) in this scenario [17]. In another scenario, the cosmic neutrinos originate from the decay of neutrons rather than pions, resulting in a flavor composition of $1: 0: 0$ (approximately 2.5:1:1 at Earth) [17]. 
Deviations from these idealized scenarios for the energy spectrum and flavor composition are likely to occur, in particular in environments with large matter or radiation densities, or large magnetic fields, see e.g. [19, 20].

\section{Detecting Neutrinos with IceCube}

The IceCube Neutrino Observatory [21] is a neutrino telescope located at the geographical South Pole in Antarctica. It consists of more than 5,000 optical sensors, installed on 86 vertical strings, buried in a cubic-kilometer of glacial ice between depths of 1,450 $\mathrm{m}$ and 2,450 m. Neutrino interactions are recorded by detecting the Cherenkov emission of secondary particles created in the interactions. Based on the arrival time and the amount of light registered in the sensors, the energy and incoming direction of the neutrino can be inferred.

The signatures that neutrinos leave in the IceCube detector can be classified in two categories. On the one hand, tracks arise from charged-current $v_{\mu}$ interactions. The muons created in such interactions can travel for several kilometers, thus leaving an elongated, track-like signature. Depending on whether the interaction occurs inside or outside the instrumented volume of the detector, we distinguish starting tracks and throughgoing tracks.

On the other hand, charged-current interactions of $v_{e}$ and $v_{\tau}$ and neutral-current interactions of all neutrinos lead to showers. The dimensions of the electromagnetic and hadronic particle showers created in these interactions are much smaller than the sensor spacing, resulting in a spherical signature. In the past, only contained showers were studied, i.e. those that start well inside the detection volume. In another contribution to this conference [12], uncontained showers that start near the edge of the detection volume are analyzed for the first time.

Charged-current interactions of $v_{\tau}$ can be identified at very high energies, $\gtrsim 1 \mathrm{PeV}$. While no $v_{\tau}$ were identified yet, a new upper limit on the $\operatorname{cosmic} v_{\tau}$ flux is presented in [11]. The limit is derived from a search for events that exhibit double pulse waveforms, i.e. a double-peak structure in the time-resolved signal recorded by the sensors. Such a signature is expected for charged-current $v_{\tau}$ interactions, where the first pulse is due to the interaction of the neutrino and the second pulse results from the decay of the tau.

\section{Analysis}

The analysis presented here is a continuation of the analysis presented in [6]. The method is summarized in the following; the reader is referred to the reference for more details.

The event samples analyzed here are listed in Table 1. Where data taking periods overlap, the event samples were separated with additional criteria, thus ensuring statistical independence of all samples. Each sample provides simulated probability density functions (PDFs) as well as experimental distributions for the observables listed in the third column of the table. PDFs are available both for background components and for the expected cosmic neutrino flux.

Background contributions to the event samples are entirely of atmospheric origin, i.e. muons and neutrinos created in air showers initiated by cosmic rays impinging on the Earth's atmosphere. The contribution of atmospheric muons is estimated by simulating air showers with the CORSIKA 


\begin{tabular}{lcccc} 
ID & Signatures & Observables & Period & References \\
\hline T1 & throughgoing tracks & energy, zenith & $2009-2010$ & {$[7]$} \\
T2 & throughgoing tracks & energy, zenith & $2010-2012$ & {$[4]$} \\
S1 & cont. showers & energy & $2008-2009$ & {$[8]$} \\
S2 & cont. showers & energy & $2009-2010$ & {$[9]$} \\
H1* & cont. showers, starting tracks & energy, zenith & $2010-2014$ & {$[1,2,10]$} \\
H2 & cont. showers, starting tracks & energy, zenith, signature & $2010-2012$ & {$[3]$} \\
DP* $^{*}$ & double pulse waveform & signature & $2011-2014$ & {$[11]$} \\
US* $^{*}$ & uncont. showers & energy & $2010-2012$ & {$[12]$} \\
\hline
\end{tabular}

Table 1: Event selections used in this analysis. Listed are the selected signatures, observables used in the analysis, and the data taking period. Event selections marked with an asterisk were newly added or extended with respect to the analysis presented in [6]. Note that only the sample of uncontained showers from reference [12], which represents a new signature previously not considered, is used here.

code [22]. Atmospheric neutrinos are divided in two categories: conventional atmospheric neutrinos originate from the decay of pions and kaons and prompt atmospheric neutrinos arise from the decay of mesons containing a charm quark. Because pions and kaons are more likely to re-interact than to decay at the energies relevant here, the energy spectrum of conventional atmospheric neutrinos is steeper than that of prompt atmospheric neutrinos, whose parent particles always decay. We use the models from Honda et al. [23] and Enberg et al. [24] to obtain a prediction of the contribution of conventional and prompt atmospheric neutrinos to the event samples, respectively. Both models were slightly modified (see [7]) to conform with recent measurements of the primary cosmic ray spectrum in the knee region, based on the composition model from [25] (known as H3a model). Furthermore, the normalizations of both components are free parameters in the analysis, denoted by $\phi_{\text {conv }}$ and $\phi_{\text {prompt }}$, respectively.

We use two different spectral hypotheses to describe the cosmic neutrino flux:

$$
\begin{array}{ll}
\text { Hypothesis A: } & \Phi_{v}=\phi \times\left(\frac{E}{100 \mathrm{TeV}}\right)^{-\gamma} \\
\text { Hypothesis B: } & \Phi_{v}=\phi \times\left(\frac{E}{100 \mathrm{TeV}}\right)^{-\gamma} \times \exp \left(-E / E_{\text {cut }}\right) .
\end{array}
$$

The first hypothesis is a simple power law, where $\phi$ denotes the flux at $100 \mathrm{TeV}$ and $\gamma$ is the spectral index. The second hypothesis allows for an exponential high-energy cut-off in addition, where the cut-off energy is denoted by $E_{\text {cut }}$. To determine the energy spectrum, we assume that the flux is composed of equal flavors at Earth. The flavor composition is then derived by varying the normalizations of all three flavors independently, assuming that their energy spectrum is identical.

Systematic uncertainties, such as the spectral index of the primary cosmic ray spectrum, or the global energy scale, are incorporated in the analysis procedure via several nuisance parameters.

The experimental data are compared to the simulated PDFs by means of a binned Poissonlikelihood analysis. The best-fit parameter values of the models are determined by maximizing the likelihood to obtain the observed distributions of observables in all samples (cf. Table 1) simultaneously. 


\section{Results}

The results of the analysis are summarized in Table 2. When no cut-off is present, the best-fit spectral index is $2.49 \pm 0.08$. If an exponential cut-off is allowed, the bestfit spectral index is $2.31_{-0.15}^{+0.14}$, and the best-fit cut-off energy is $\left(2.7_{-1.4}^{+7.7}\right) \mathrm{PeV}$. These measurements are valid in the energy range $27 \mathrm{TeV}-2 \mathrm{PeV}$ (hypothesis $\mathrm{A}$ ) and $22 \mathrm{TeV}-5.3 \mathrm{PeV}$ (hypothesis B). The hypothesis with a cut-off is slightly preferred, although with a significance of only $1.2 \sigma(p=12 \%)$. Both models describe the data reasonably well. On the other hand, an unbroken power law spectrum with spectral index $\gamma=2$ can be excluded with a significance of $4.6 \sigma(p=0.00018 \%)$.

The correlation between the spectral index $\gamma$ and the cut-off energy $E_{\text {cut }}$ is visualized in fig. 1(a). Figure 1(b) shows the best-fit spectrum for both hypotheses together with a differential model that extracts the cosmic neutrino flux in separate energy intervals.

Results on the flavor composition are presented in table 3 and fig. 2. For both spectral hypotheses, the flux consists of $v_{e}$ and $v_{\mu}$ in approximately equal parts at the best fit. However, compositions expected for pion-decay sources $(1: 2: 0$ at source) and muon-damped sources $(0: 1: 0$ at source) are still compatible with our data. On the other hand, a flux composed purely of electron neutrinos at the source is excluded with a significance of $3.7 \sigma(p=0.012 \%)$.

The derived constraints are improved, but largely similar with respect to those of the previous analysis [6]. While the hypothesis with an exponential cut-off is now marginally preferred, no firm conclusion can be drawn at this point.

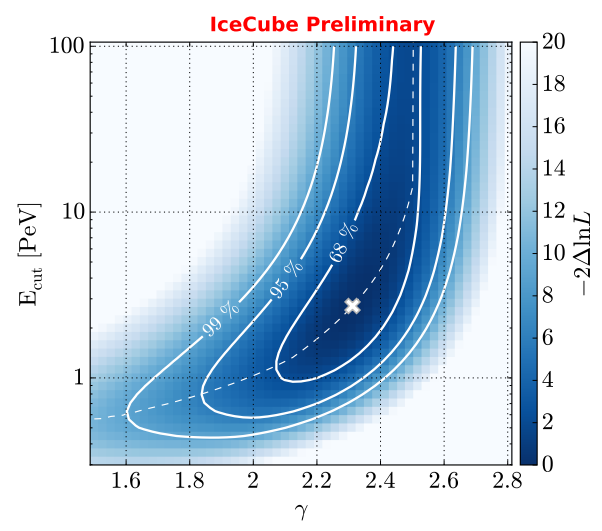

(a)

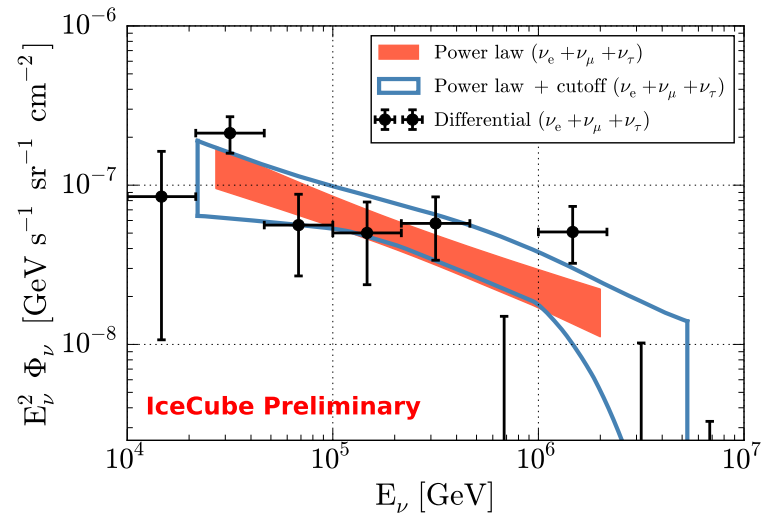

(b)

Figure 1: Results on the energy spectrum. (a) Profile likelihood scan of parameters $\gamma$ and $E_{\text {cut }}$. The best fit is marked with ' $\times$ '. The dashed line shows the conditional best-fit value of $E_{\text {cut }}$ for each value of $\gamma$. (b) Energy spectrum of the cosmic neutrino flux. Shown are the spectra allowed at $68 \%$ C.L. for hypothesis A (power law) and hypothesis B (power law + cutoff). In addition, the strength of the cosmic neutrino flux in separate energy intervals is shown (differential). 
The impact of the newly added tau search event sample (DP, cf. table 1) is mainly visible in the flavor composition fit, see fig. 2. In contrast, the new event sample of uncontained showers (US) and the extended event sample of analysis H1 mainly affect the energy spectrum and

\begin{tabular}{cccc} 
Param. & Unit & Hyp. A & Hyp. B \\
\hline$\phi_{e}$ & $10^{-18} \mathrm{GeV}^{-1} \mathrm{~s}^{-1} \mathrm{sr}^{-1} \mathrm{~cm}^{-2}$ & $3.2_{-1.5}^{+0.7}$ & $3.6_{-1.7}^{+0.8}$ \\
$\phi_{\mu}$ & $10^{-18} \mathrm{GeV}^{-1} \mathrm{~s}^{-1} \mathrm{sr}^{-1} \mathrm{~cm}^{-2}$ & $3.3_{-0.6}^{+0.7}$ & $3.7_{-0.7}^{+0.8}$ \\
$\phi_{\tau}$ & $10^{-18} \mathrm{GeV}^{-1} \mathrm{~s}^{-1} \mathrm{sr}^{-1} \mathrm{~cm}^{-2}$ & $0.0_{-0.0}^{+2.4}$ & $0.0_{-0.0}^{+2.6}$ \\
$\gamma$ & - & $2.53_{-0.08}^{+0.08}$ & $2.35_{-0.15}^{+0.14}$ \\
$E_{\text {cut }}$ & $\mathrm{PeV}$ & - & $2.7_{-1.4}^{+7.5}$ \\
\hline$-2 \Delta \ln L$ & & +1.69 & 0 \\
\hline
\end{tabular}

Table 3: Best-fit results for the flavor composition fit. The quoted uncertainties are at $1 \sigma$ confidence level.

contribute to the slight preference of an exponential cut-off. The samples DP and US represent new event signatures that were previously not considered in the analysis. Almost all of the event selections can be applied to new data that are already recorded. The resulting expected sensitivity to the energy spectrum and flavor composition is investigated in the following section.

\section{Projected Sensitivities}

In order to derive the future sensitivity of the IceCube detector to the properties of the cosmic neutrino flux, we use a prototype analysis that is based on the event selections of samples T2, H2, DP, and US (cf. Table 1). We weight the simulated cosmic neutrino flux to the current best-fit energy spectrum of hypothesis A or B (cf. previous section) and

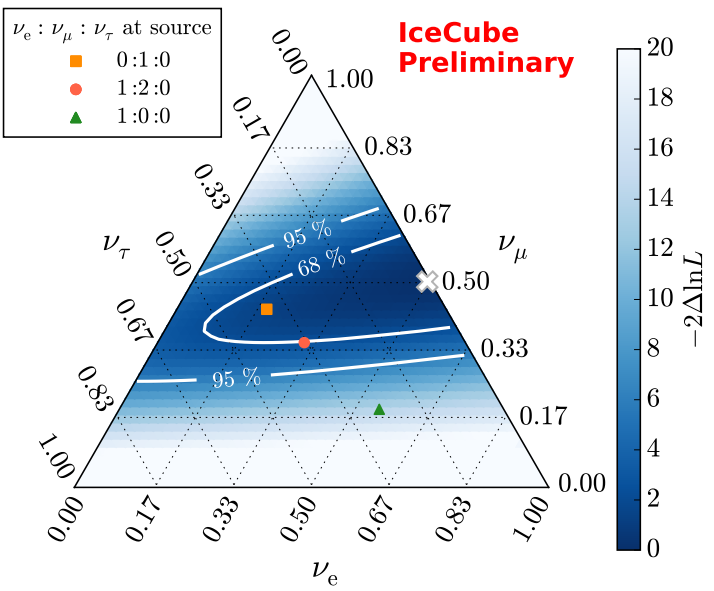

Figure 2: Results on the flavor composition, using hypothesis B for the energy spectrum. Each point on the triangle corresponds to a ratio $v_{e}: v_{\mu}: v_{\tau}$ as measured at Earth. The best fit is marked with ' $x$ '. Compositions expected for three different source scenarios are indicated. scale the expected signal up to mimic the collection of additional data. For the conventional and prompt atmospheric neutrino flux, we assume a flux at the level of the predictions by Honda et al. [23] and Enberg et al. [24], respectively. The sensitivity is then derived using the approach described in [26].

The projected sensitivity to the energy spectrum is illustrated in fig. 3(a), where we focus on the sensitivity to the presence of an exponential high-energy cut-off to the spectrum. The two large panels show expected limits on the energy of such an exponential cut-off, where the current best-fit spectrum of hypothesis A and B is assumed to be the true spectrum in the top and bottom panel, respectively. If no cut-off is present, the expected lower limit with 10 years of full detector data is 6.7 PeV at $2 \sigma$ confidence, i.e. well above the current best-fit value of $2.7 \mathrm{PeV}$. On the other hand, for a true cut-off energy at the current best fit, the non-existence of an exponential cut-off can be rejected with a significance of $\sim 3 \sigma$ with 10 years of data. Note that a single isotropic cosmic neutrino flux is assumed in all cases. 
Finally, to illustrate our future sensitivity to the flavor composition of the cosmic neutrino flux, we show the median expected constraints for 10 years of full detector data in fig. 3(b). Here, we assume an energy spectrum matching the current best fit of hypothesis B and that the cosmic neutrino flux consists of equal flavors. Although we included a search for $v_{\tau}$ signatures in this analysis for the first time, a degeneracy with respect to the $v_{e} / v_{\tau}$ fraction remains, resulting in the elongated shape of the contours. However, the ability to distinguish between different source scenarios is largely orthogonal to this degeneracy, and thus not affected.

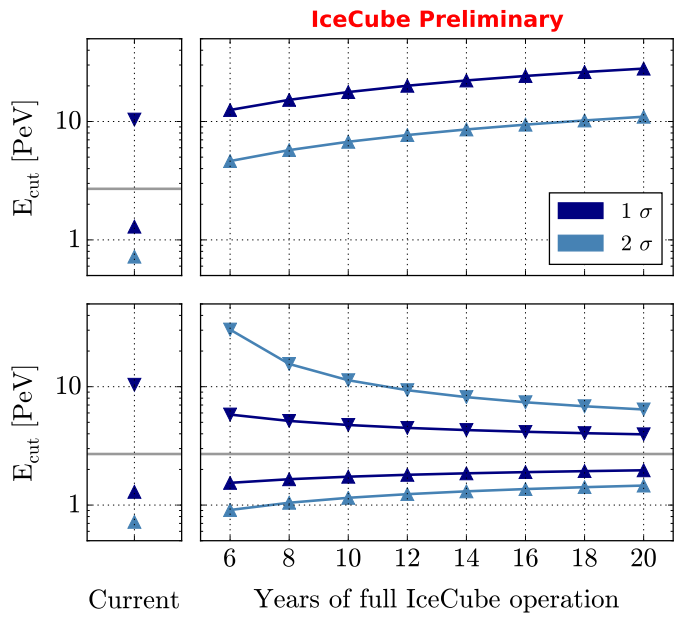

(a)

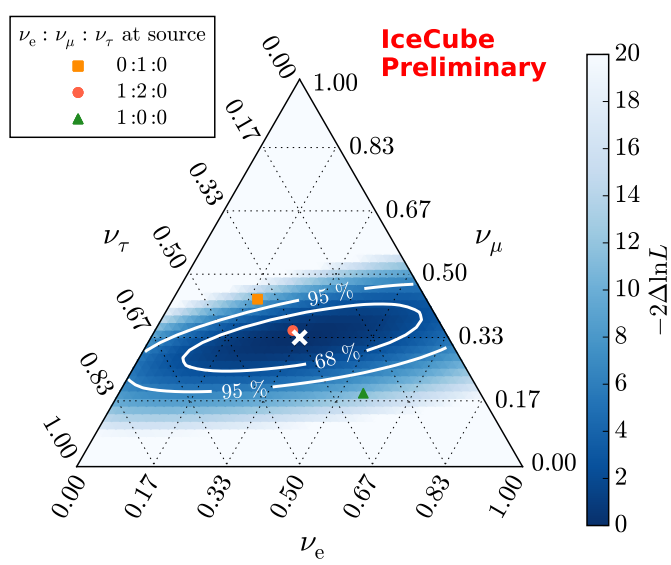

(b)

Figure 3: (a) Current (small panels) and projected (large panels) constraints on the energy of an exponential cut-off to the spectrum. The two small panels show the best-fit result of hypothesis B. In the top (bottom) right panel, the best-fit spectrum of hypothesis A (B) is assumed to be true. Dark and light blue points indicate median $1 \sigma$ and $2 \sigma$ limits, respectively. (b) Projected sensitivity to the flavor composition, for 10 years of full detector data. The white ' $\times$ ' marks the assumed true flavor composition of $v_{e}: v_{\mu}: v_{\tau}=1: 1: 1$.

\section{Summary}

We have presented a continuation of the combined likelihood analysis of the diffuse cosmic neutrino flux presented in [6]. We find that the energy spectrum of the cosmic neutrino flux is well described by an unbroken power law, with a best-fit spectral index of $-2.49 \pm 0.08$. This corresponds to a rejection of an unbroken power law with index -2 with $4.6 \sigma$ significance $(p=$ $0.00018 \%)$. While the analysis slightly favors a harder power law $\left(E^{-2.31 \pm 0.15}\right)$ with an exponential cut-off at $\left(2.7_{-1.4}^{+7.7}\right) \mathrm{PeV}$ over an unbroken power law, the statistical significance of $1.2 \sigma(p=12 \%)$ is too low to draw any conclusion. However, we have shown that the presence of a cut-off can likely be determined with additional data in the foreseeable future. Other, more complex spectral shapes are also possible, although currently not required to describe the data.

The flavor composition of the cosmic neutrino flux is compatible with standard scenarios for the neutrino production at the sources. However, a neutron-decay dominated scenario, in which only electron neutrinos are produced at the sources, can be ruled out with $3.7 \sigma$ significance $(p=0.012 \%)$. A projection of our sensitivity to the flavor composition has shown that while a 
degeneracy with respect to the $v_{e} / v_{\tau}$ fraction remains, a distinction between more source scenarios might be possible.

\section{References}

[1] M. G. Aartsen et al. (IceCube Collaboration), Science 342 (2013) 1242856. [arXiv:1311.5238].

[2] M. G. Aartsen et al. (IceCube Collaboration), Phys. Rev. Lett. 113 (2014) 101101. [arXiv:1405.5303].

[3] M. G. Aartsen et al. (IceCube Collaboration), Phys. Rev. D 91 (2015) 022001. [arXiv:1410.1749].

[4] M. G. Aartsen et al. (IceCube Collaboration), Evidence for Astrophysical Muon Neutrinos from the Northern Sky with IceCube, submitted to Phys. Rev. Lett. (2015). [arXiv:1507.04005].

[5] M. G. Aartsen et al. (IceCube Collaboration), Phys. Rev. Lett. 114 (2015) 171102. [arXiv:1502.03376].

[6] M. G. Aartsen et al. (IceCube Collaboration), A combined maximum-likelihood analysis of the high-energy astrophysical neutrino flux measured with IceCube, accepted by Astrophys. J. (2015). [arXiv:1507.03991].

[7] M. G. Aartsen et al. (IceCube Collaboration), Phys. Rev. D 89 (2014) 062007. [arXiv:1311.7048].

[8] M. G. Aartsen et al. (IceCube Collaboration), Phys. Rev. D 89 (2014) 102001. [arXiv:1312.0104].

[9] A. Schönwald et al. for the IceCube Collaboration, Search for diffuse astrophysical neutrinos with cascade events in the IceCube-59 detector, in Proc. 33rd ICRC, 2013. arXiv:1309.7003.

[10] M. G. Aartsen et al. (IceCube Collaboration), PoS(ICRC2015)1081, these proceedings.

[11] M. G. Aartsen et al. (IceCube Collaboration), PoS(ICRC2015)1071, these proceedings.

[12] M. G. Aartsen et al. (IceCube Collaboration), PoS(ICRC2015)1109, these proceedings.

[13] T. K. Gaisser, F. Halzen, and T. Stanev, Phys. Rep. 258 (1995) 173-236.

[14] J. G. Learned and K. Mannheim, Annu. Rev. Nucl. Part. S. 50 (2000) 679-749.

[15] J. K. Becker, Phys. Rep. 458 (2008) 173-246. [arXiv:0710.1557].

[16] P. Lipari, M. Lusignoli, and D. Meloni, Phys. Rev. D 75 (2007) 123005. [arXiv:0704.0718].

[17] S. Choubey and W. Rodejohann, Phys. Rev. D 80 (2009) 113006. [arXiv:0909.1219].

[18] T. K. Gaisser, Cosmic Rays and Particle Physics. Cambridge University Press, Cambridge, 1990.

[19] T. Kashti and E. Waxman, Phys. Rev. Lett. 95 (2005) 181101. [arXiv:astro-ph/0507599].

[20] W. Winter, J. Becker Tjus, and S. R. Klein, Astron. Astrophys. 569 (2014) A58. [arXiv:1403.0574].

[21] T. K. Gaisser and F. Halzen, Annu. Rev. Nucl. Part. S. 64 (2014) 101-123.

[22] D. Heck, J. Knapp, J. N. Capdevielle, G. Schatz, and T. Thouw, CORSIKA: a Monte Carlo code to simulate extensive air showers, Tech. Rep. FZKA 6019, Forschungszentrum Karlsruhe, 1998.

[23] M. Honda, T. Kajita, K. Kasahara, S. Midorikawa, and T. Sanuki, Phys. Rev. D 75 (2007) 043006. [arXiv:astro-ph/0611418].

[24] R. Enberg, M. H. Reno, and I. Sarcevic, Phys. Rev. D 78 (2008) 043005. [arXiv:0806.0418].

[25] T. K. Gaisser, Astropart. Phys. 35 (2012) 801-806. [arXiv:1111.6675].

[26] G. Cowan, K. Cranmer, E. Gross, and O. Vitells, Eur. Phys. J. C 71 (2011) 1554. [arXiv:1007.1727]. 